\title{
Laser pointer induced foveal injury
}

\begin{abstract}
17 years old male with no past medical or ophthalmic history. History of exposure to laser pointer by friend for a few seconds at close distance. Subsequent decrease in vision in weeks time with complaints of central scotoma. Power of laser unknown in mW.
\end{abstract}

Keywords: foveal trauma, laser pointing devices
Volume 4 Issue 5 - 2016

\section{Waseem Qureshi}

Department of Ophthalmology, Great Western Hospital, UK department, Great Western Hospital, 59 East Wichel Way, Swindon, SNI 7AD, UK, Tel 0044798642068I. Emailwazeem77@hotmail.com

Received: July 19, 2016 | Published: August 22, 2016

\section{Introduction}

LASERS, light amplification by simulated emission of radiation can be used in different ways. They actually produce a beam of light that is single direction, coherent and of a single colour wavelength. It can concentrate most of its radiant power, over large distances to a certain point. Lasers are being used in different daily used devices. Some of them are being used by adults only while most of them form part of devices which are used by adults as well as children. Simple use devices include scanners, printers and even mouse used in computer.

The capacity of these devices to cause damage to the eye is a grey area which needs careful consideration. The Manufacturers warn regarding a few major concerns without the background knowledge of the potential threats that prolonged usage can invoke on a potentially at risk fragile minds and eyes of an age group which are at risk of being exposed beyond any calculated or estimated risk. The different colour laser are just a difference in wavelength, the area of absorption or extent of absorption can be different but it's more to do with the focusing of the beam on the area of absorption which can be potentially threatening.

\section{Material and methods}

\section{History}

17 years old male with no past medical or ophthalmic history. He had exposed to laser pointer by friend for a few seconds at close distance, subsequently he noticed a decreased vision in following weeks with central scotoma. Power of laser unknown in $\mathrm{mW}$. No history of refractive error or contact lens wear was noted.

\section{Examination}

$6 / 6$ in right eye, 6/12 in left eye. Intraocular pressures are normal in both eyes. Anterior segment normal. Colour vision plates none read. Confrontational visual field tests indicated no obvious defect. Amsler grid showed a small area of distortion near left macula.

\section{Results}

Fundus examination revealed retinal pigment epithelial changes on the fovea confirmed by OCT scan which is corresponding with the central scotoma on Amsler grid monitoring. Follow-up examinations revealed reduction in the area of RPE (retinal pigment epithelial hypertrophy) with no or little improvement of visual acuity measured by standardized Snellen or log MAR equivalent. There was no improvement in the area of central scotoma. The mystery remained on the power or class of laser pointer used. The RPE hypertrophy did not warrant any treatment as there was minimal evidence of inflammation and or fluid on the OCT scan. The RPE changes explained the area of scotoma and resolution in the thickness mapping of the subfoveal changes. After follow up of three months the patient who was a Turkish immigrant was lost to follow up (Figure 1-4).

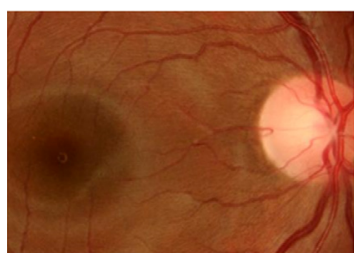

Figure I Right fundus photograph, which was normal.

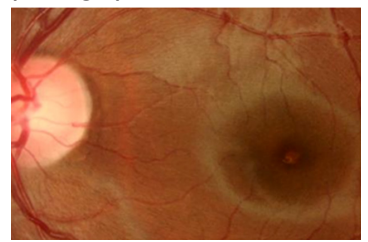

Figure 2 Left fundus photograph showing retinal pigment epithelial changes.

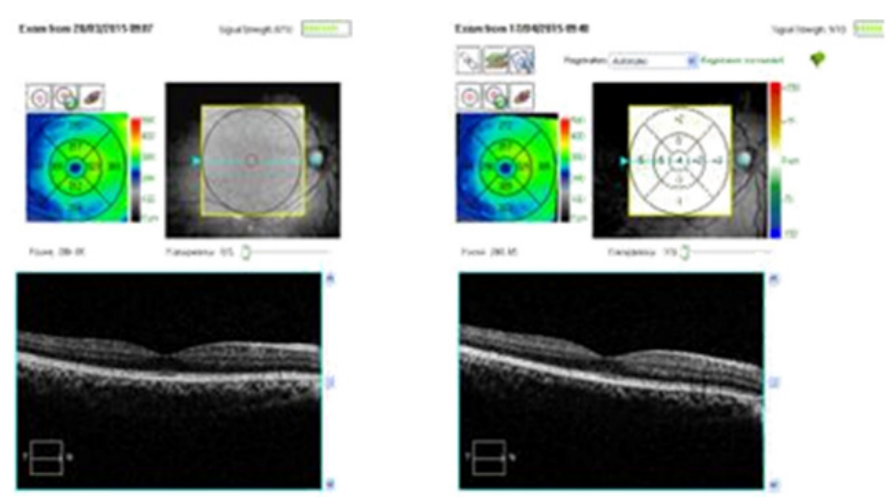

Figure 3 OCT right eye with no significant changes. 

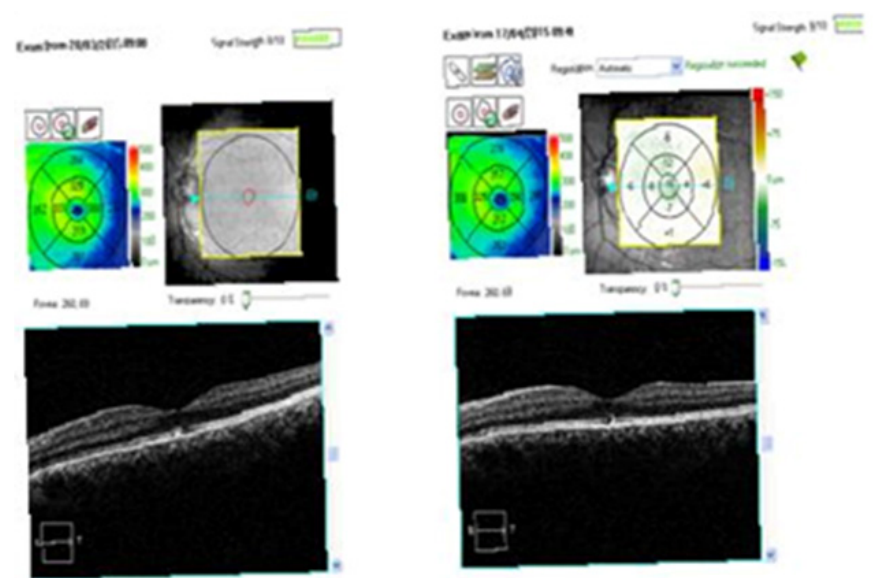

Figure 4 OCT left eye showing retinal pigment epithelial changes.

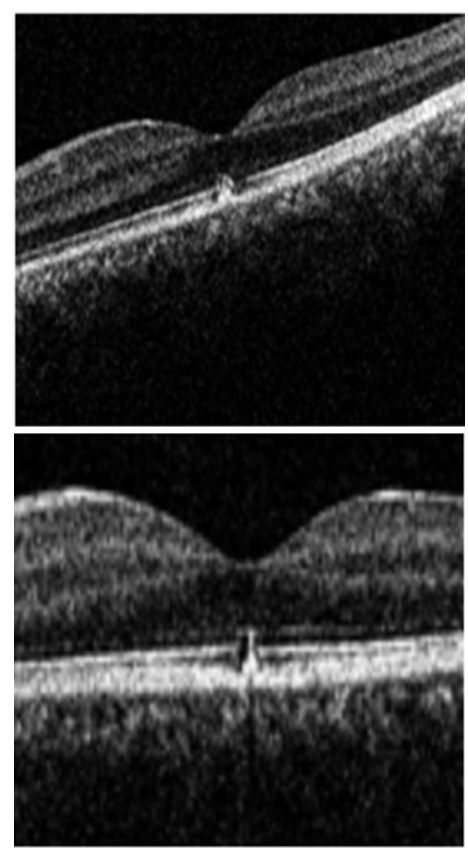

Figure 4A \& 4B Magnified views of left OCT.

\section{Discussion}

The everyday usage of these devices has made them a potential source of threat. The quantitative risk assessment depends not only on the nature of the risk but also the frequency of the exposure which makes it more likely to cause damage. The imaging studies to date are inconclusive in certain circumstances to actually quantify or even in certain cases identify the nature or extent of the damage. ${ }^{1-3}$ There are only a few studies regarding the harmful effects of red and even less green lasers. Retinal lesions induced by laser pointers include drusen like deposits; ring shaped hypo pigmentation around fovea, granular changes of fovea, sub retinal hemorrhage, vitreous or chorioretinal hemorrhage, retinal edema, choroidal neovascularization and scarring due to extensive retinal pigment epithelial changes. ${ }^{4}$ Barkana and Belkin, in their paper have concluded that several factors contribute to laser-related retinal damage. Factors which are laser-related including wavelength of the radiation, energy and pulse duration of the beam. Other factors are related to the patients like size of the pupil, degree of retinal pigmentation, closeness to the fovea, refractive status, more in emmetropes, alarmingly dependent on the focus of the beam. ${ }^{5}$
Various experimental studies have taken into account the clinical and histopathological changes that are induced by the laser pointers in patients which were undergoing enucleation for melanoma, showed that wave lengths $490-575 \mathrm{~nm}$, being green, are more damaging due to the absorption at the fovea compared to the $635-750 \mathrm{~nm}$, being red colour. In adults the blink reflex is a very protective response that limits the exposure time of the laser to 0.25 seconds; also pupillary response and aversion do the same. Children usually infants display an abnormal staring response to the laser pointers for a prolonged period of time without averting or blinking the eye. Some studies show that corticosteroids can be effective in treating these injuries. This is of undetermined results and results which cannot be standardized. The visual improvement could be unlikely or minimal based upon the objective visual assessment scales if without treatment. The visual acuity ranges in some studies is $20 / 20$ to $20 / 60$ improvement in different lesions depending on the size and location of the initial lesion from the fovea.

The various safety guidelines are constantly being revised regarding these devices. But the fact remains that some of these devices are easily available online and are a potent hazard in hands of children. The various criteria for grading these devices and the their power in terms $\mathrm{mW}$ is a good way to make only low power laser devices which can limit the chances of damage to the fovea but only to adult population. This report emphasizes the importance of cautious and appropriate use of laser pointer devices, need for more robust safety guidelines and laws because of the potential vision-threatening hazards induced by mishandling of these devices.

\section{Conclusion}

Fact remained the online and retail availability of such devices and the usage in hands of children or unalarmed individual can be devastating if focused directly on the macula, in a dilated pupil can be potentially devastating.

\section{Acknowledgments}

None.

\section{Conflicts of interest}

The authors declare there are no conflicts of interest.

\section{Funding}

None.

\section{References}

1. Robertson DM, Lim TH, Salomao DR, et al. Laser pointers and the human eye: a clinicopathologic study. Arch Ophthalmol. 2000;118(12):16861691.

2. Mensah E, Vafidis G, Marshall J. Laser pointers: the facts, media hype, and hysteria. Lancet. 1998;351(9111):1291.

3. Van Norren D, Keunen JE, Vos JJ. The laser pointer: no demonstrated danger to the eyes. Ned Tijdschr Geneeskd. 1998;142(36):1979-1982.

4. Turaka K, Bryan JS, Gordon AJ, et al. Laser pointer induced macular damage: case report and mini review. Int Ophthalmol. 2012;32(3):293297.

5. Barkana Y, Belkin M. Laser eye injuries. Surv Ophthalmol. 2000;44(6):459-478. 